\title{
Erstes Antidot gegen ein NOAK in klinischer Studie untersucht
}

Fragestellung: Kann ein monoklonaler Antikörper gegen Dabigatran bei Patienten, die unter Dabigatran bluten oder einen dringenden operativen Eingriff benötigen, die Gerinnungsparameter normalisieren?

Hintergrund: Dabigatran ist seit fünf Jahren zur Schlaganfallprävention bei Vorhofflimmern sowie zur Prophylaxe und Therapie der tiefen Beinvenenthrombose zugelassen. Bis jetzt gab es kein spezifisches Antidot, um Patienten, die Dabigatran einnahmen, in spezifischen Notfallsituationen zu behandeln. Deshalb entwickelten Grundlagenforscher bei Boehringer Ingelheim einen monoklonalen Antikörper, der mit hoher Affinität Dabigatran bindet. Bei jungen und älteren Gesunden sowie bei Patienten mit Nierenfunktionsstörungen führte Idarucizumab zu rascher und kompletter Normalisierung der durch Dabigatran beeinflussten Gerinnungsparameter. Das war Anlass, eine prospektive Studie bei Patienten mit Blutungen unter Dabigatran durchzuführen oder bei Patienten, bei denen ein dringender operativer und diagnostischer Eingriff notwendig ist.

Patienten und Methodik: Die RE-VERSE-AD-Studie ist eine prospektive Kohorten-Studie in die 300 Patienten in 400 Zentren und 38 Ländern eingeschlossen werden sollen. Die Patienten werden in zwei Gruppen eingeteilt: Bei der ersten

Pollack CV Jr, Reilly PA, Eikelboom J et al. Idarucizumab for dabigatran reversal. N Engl J Med 2015; 373: $511-20$ Gruppe kommt es unter Dabigatran $\mathrm{zu}$ schwerwiegenden Blutungskomplikationen und die zweite Gruppe benötigt ein normalisiertes Gerin- nungssystem wegen einer dringend anstehenden Operation oder einer invasiven Prozedur. In der Publikation werden die Ergebnisse der ersten 90 Patienten referiert, wobei der primäre Endpunkt die Normalisierung der verdünnten Thrombinzeit und der erhöhten Ecarin-Clotting-Zeit ist.

Ergebnisse: Zwischen Juni 2014 und Februar 2015 wurden 90 Patienten behandelt, dabei handelte es sich um 51 Patienten mit schwerwiegenden Blutungen und 39 Patienten die einen raschen Eingriff benötigten. Die Patienten waren im Mittel 76 Jahre alt. Ein Drittel der Patienten hatte die hohe Dosis und zwei Drittel die niedrige Dosis von Dabigatran erhalten. Bei fast allen Patienten erfolgte die Behandlung mit Dabigatran aufgrund von Vorhofflimmern. Die mediane Zeit seit der letzten Einnahme von Dabigatran betrug im Mittel 15 Stunden. Bei 76\% der Patienten war die verdünnte Thrombinzeit vor der Behandlung erhöht und bei $90 \%$ die Ecarin-Clotting-Zeit. $20 \%$ der Blutungen waren intrakraniell, $10 \%$ traumatisch, $22 \%$ gastrointestinal und $12 \%$ andere.

Idarucizumab wurde in zwei Infusionen mit jeweils $2,5 \mathrm{~g}$ gegeben. Innerhalb weniger Minuten kam es bei $98 \%$ der Patienten zu einer Normalisierung der Gerinnungswerte. Von $36 \mathrm{~Pa}$ tienten in der Gruppe, die operiert werden mussten, berichteten die Chirurgen, dass bei 33 Patienten die intraoperative Hämostase erhalten war. Nur bei einem Patienten kam es innerhalb von 72 Stunden zu einem arteriothrombotischen Ereignis, wobei dieser Patient nicht erneut antikoaguliert worden war.

Schlussfolgerung: Idarucizumab kann innerhalb kurzer Zeit die Wirkung von Dabigatran aufheben.

\section{- Kommentar von Hans-Christoph Diener, Essen}

\section{Prospektive Register nach der Zulassung sind wünschenswert}

Die Entwicklung und klinische Erprobung eines spezifischen Antidots gegen Antikoagulantien ist von großer Bedeutung für die klinische Praxis. Zwar sind schwerwiegende Blutungskomplikationen unter Dabigatran relativ selten und können auch mit Prothrombinkomplex oder Fresh Frozen Plasma behandelt werden. Im klinischen Alltag ist allerdings ein spezifisches Antidot sehr viel hilfreicher und wirksamer, da hier innerhalb von Minuten die Gerinnungswerte normalisiert werden. Zunächst bleibt abzuwarten, ob sich die Ergebnisse der ersten 90 Patienten bis zum Ende der Studie mit 300 Patienten replizieren lassen. Die Ergebnisse der ersten 90 Patienten wurden bei den Zulassungsbehörden eingereicht und es ist möglich, dass Idarucizumab bereits im Herbst oder Winter 2015 zugelassen wird. Wünschenswert wären dann prospektive Register, die überprüfen, ob sich auch in größeren Popu- lationen ein klinischer Effekt bezüglich Überlebensraten zeigt. Eine randomisierte Studie wäre gemessen an der guten Wirksamkeit von Idarucizumab ethisch nicht vertretbar.

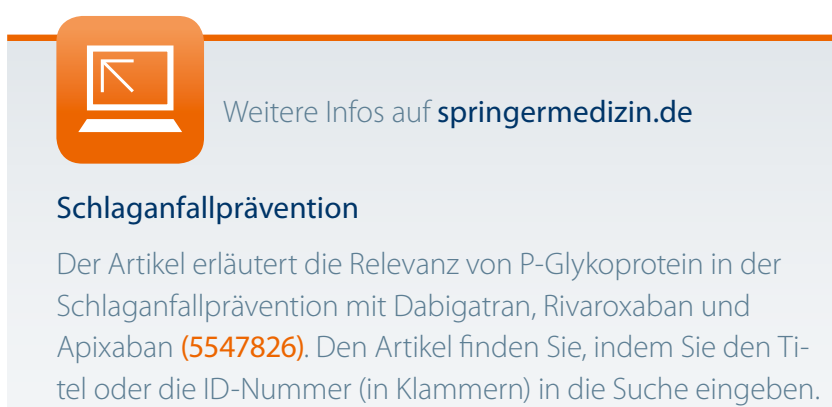

